\title{
Trivium
}

Revue franco-allemande de sciences humaines et sociales - Deutsch-französische Zeitschrift für Geistesund Sozialwissenschaften

9| 2011

Vitesse et existence. La multiplicité des temps historiques

\section{Das Gefühl der Beschleunigung der modernen Geschichte: Bausteine für eine Geschichte}

\author{
Alexandre Escudier \\ Traducteur : Achim Russer
}

\section{OpenEdition}

Journals

Édition électronique

URL : http://journals.openedition.org/trivium/4034

ISSN : 1963-1820

Éditeur

Les éditions de la Maison des sciences de l'Homme

Référence électronique

Alexandre Escudier, « Das Gefühl der Beschleunigung der modernen Geschichte: Bausteine für eine Geschichte », Trivium [Online], 9 | 2011, online erschienen am 30 November 2011, abgerufen am 07 September 2020. URL : http://journals.openedition.org/trivium/4034

Ce document a été généré automatiquement le 7 septembre 2020

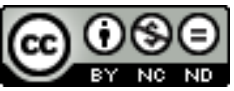

Les contenus des la revue Trivium sont mis à disposition selon les termes de la Licence Creative Commons Attribution - Pas d'Utilisation Commerciale - Pas de Modification 4.0 International. 


\title{
Das Gefühl der Beschleunigung der modernen Geschichte: Bausteine für eine Geschichte
}

\author{
Alexandre Escudier
}

Traduction : Achim Russer

\section{NOTE DE L'ÉDITEUR}

Wir danken Alexandre Escudier und der Zeitschrift Esprit für die freundliche Genehmigung, diesen Artikel zu übersetzen.

Reinhart Koselleck zum Gedenken

»Bald wird man streng abgeschirmte Klöster bauen müssen, in die weder die Wellen noch die

Blätter eindringen; in denen die Ignoranz jeglicher Politik bewahrt und gepflegt wird. Dort wird man Verachtung empfinden für Geschwindigkeit, für große Zahlen, für die Massen-, die Überraschungs-, Wiederholungs-, Neuheits- und Leichtgläubigkeitseffekte. Dorthin wird man gehen müssen an manchen Tagen und durch die Gitterstäbe hindurch einige Exemplare

von freien Menschen betrachten. ${ }^{1}$

Die »Beschleunigung der Geschichte« gehört gewiss $\mathrm{zu}$ jenen zeitgenössischen Schlagwörtern, die ebenso selten analysiert wie häufig zitiert werden. Von Reinhart Koselleck $^{2}$ und neuerdings Peter Borscheid ${ }^{3}$ und Hartmut Rosa ${ }^{4}$ einmal abgesehen, haben nur wenige Autoren versucht, das Phänomen in seiner ganzen Komplexität zu erkunden. Das Schlagwort nährt somit die essayistische und journalistische Prosa, ohne 
es jemals zu einem wohldefinierten Begriff oder einem Idealtypus mit heuristischen Qualitäten zu bringen. Die Gründe dafür sind leicht nachvollziehbar: Näher besehen zeigt sich nämlich, dass das Problem der »Beschleunigung der Geschichte» derart unterschiedliche und heterogene Wirklichkeiten einschließt, dass man sich zu Recht die Frage stellt, ob die darin einbegriffenen Phänomene und Diagnosen überhaupt einen wirklichen, als solchen objektivierbaren "Gegenstand" darstellen. Womit gemeint ist: objektivierbar von welchem Korpus, von welcher Periode aus, für welchen Raum und für welche Typen sozialer, politischer und kultureller Milieus?

2 Das Thema der "Beschleunigung der Geschichte" verweist ebensowohl auf die subjektive Seite der sozialen Zeiterfahrung wie auf die objektive Seite der dynamischen Gesellschaftsstrukturen. Insofern begreift es nichts weniger ein als die Geschichte aller Wandlungsfaktoren der Modernität seit dem 18. Jahrhundert: den technischen, den sozialen, den kulturellen und auch, oder vielleicht vor allem, den symbolischen Wandel. Er verweist damit gleichermaßen auf die Technikgeschichte (innerhalb deren die Beschleunigung des Güter- und Personentransports deutlich zu unterscheiden ist von der Beschleunigung der Übermittlung schlichter Informationen) wie auf die Geschichte des strukturellen Wandels der wirtschaftlichen und sozialen Organisation der Moderne und der all diesen Mutationen entsprechenden politischen und kulturellen Geschichte.

3 Von vornherein ist daher auf eine außerordentliche methodologische Schwierigkeit hinzuweisen, deren Erwähnung weit mehr ist als eine bloße rhetorische Vorkehrung: auf die Schwierigkeit, einen (kausalen) Zusammenhang zwischen konkreten Phänomenen materieller Beschleunigung der modernen Gesellschaften und diversen historischen Semantiken herzustellen, die auf mehr oder weniger konfuse Weise Beschleunigungsdiagnosen der Geschichte seit dem 18. Jahrhundert formulieren. Wie lassen sich die Verkettungsebenen zwischen der objektiven (materiellen) Seite und der subjektiven (wahrnehmungsmäßigen, vorstellungsgemäßen, kurz: gefühlshaften) Seite des Phänomens der so genannten "Beschleunigung der Geschichte" eigentlich historisch fassen? Die Erklärung stößt hier auf eine Quadratur des Kreises, die man zu lösen versuchen kann, indem man die unterschiedlichen empirischen Materialien, die hier vorliegen, dem Koselleckschen Doppelregister entsprechend in »Erfahrungsraum « (objektive Seite) und "Erwartungshorizont" (subjektive Seite) aufteilt - d.h. entsprechend dem Doppelregister passiver Affektion und spontaner Aktivität der historischen Subjekte (also der großen Husserlschen Sonderung von »Retention« und "Protention«, die selbst wiederum die dem hier zitierten Koselleckschen Kategorienpaar implizite Augustinische Thematik der distentio animi aufgreift).

4 In der folgenden Darstellung werde ich mich auf die Herausarbeitung derjenigen Punkte beschränken, die mir - vom millenaristischen Thema der Beschleunigung der Endzeit bis hin zu den zeitgenössischen Diagnosen der Beschleunigung der Geschichte und den gegenwärtigen utopischen Wünschen nach Entschleunigung - als Kernpunkte dieser ganzen Angelegenheit erscheinen.

\section{„Die Beschleunigung der Endzeit« vor der »Beschleunigung der Geschichte»}

Die Thematik der Beschleunigung der Geschichte präzis erfassen heißt zunächst einmal, sie von einer im Okzident sehr viel älteren Semantik abgrenzen: der der 
»Beschleunigung der Endzeit«. Von den ersten millenaristischen Interpretationen einiger Schlüsselstellen der Bibel angefangen bis hin zum Beginn des 19. Jahrhunderts entstand eine Unzahl zunächst eschatologischer und sodann »chiliastischer « (d.h. millenaristischer) Diskurse ${ }^{5}$, die - ausgehend von den alttestamentarischen Prophezeiungen und vor allem der Geheimen offenbarung des Johannes - verkünden, dass das Ende der Zeiten im Sturmschritt herannahe. Die historische und kategoriale Unterscheidung zwischen diesen beiden unterschiedlichen Erwartungshaltungen - die »Beschleunigung der Endzeit» einerseits, die »der Geschichte« andererseits - scheint mir umso entscheidender, als die mit der ersten "Beschleunigung" verbundenen Diskurse $^{6}$ dem Gedächtnishorizont unseres säkularisierten historischen Bewusstseins heute weitgehend entschwunden sind und die Verbindung zwischen den beiden Phänomenreihen im 18. und frühen 19. Jahrhunderten der Aufmerksamkeit der Experten überwiegend entging.

6 Das Spezifische dieses Beschleunigungsdenkens liegt darin, dass die Geschichte im Wesentlichen bereits geschrieben ist. Wir kennen den Anfang (die Erschaffung der Welt), die Mitte (die Fleischwerdung Christi) und das Ende (die Parusie), aber wir kennen weder den genauen Zeitpunkt dieses Endes (des Endes der Zeiten, der Wiederkehr Christi und der Auferstehung der Toten $)^{7}$ noch wissen wir, ob ihr auf Erden ein Millenium, d.h. eine vorparadiesische Friedensperiode von tausend Jahren vorangeht, die für die einen der ersten Auferstehung der Heiligen und der Seelen, für die anderen (die darin Joachim von Fiore folgen) einem dritten, innerweltlichen Zeitalter der heiligen Geschichte entspricht: dem Zeitalter des Geistes. ${ }^{8}$

7 Ausgehend von der biblischen Angabe, die Welt sei 6000 Jahre alt, legten manche Autoren im 18. und im frühen 19. Jahrhundert ihr Ende auf das Jahr 1836 oder auf 1816 (und zwar auf den 18. Juni) fest. Die Erweckungssekte des schwäbischen Protestantismus, gestützt vor allem auf den Erfolg der chiliastischen Thesen des Johann Albrecht Bengel ${ }^{9}$ und die Schriften Johann Heinrich Jung-Stillings, eines renommierten Kameralisten ${ }^{10}$, stellt ein in Deutschland allbekanntes Beispiel für diese Spekulationen über das voraussichtliche Datum des Endes dar.

8 Aber im Grunde wissen die Menschen nichts darüber. ${ }^{11}$ »Denn tausend Jahre sind vor dir wie der Tag, der gestern vergangen ist, und wie eine Nachtwache«, heißt es im 90. Psalm. ${ }^{12}$ Folglichmuss man sich stets bereithalten ${ }^{13}$, denn das Ende der Zeiten kann jederzeit eintreffen und Christus zurückkehren »zu einer Stunde, da ihr nicht meinet«. ${ }^{14}$ Die Beschleunigung, von der in diesem Rahmen die Rede ist, ist die der Überstürzung der Ereignisse infolge der Ankunft des Antichrist, der just vor der Wiederkunft Christi auftaucht, kurz bevor die Welt durch die zweite Auferstehung (die der Körper) gerettet wird. Die Beschleunigung oder Verkürzung der Zeit blieb mithin sehr lange eine Kategorie der Erwartung. Dies ermöglichte zwei Grundhaltungen:

9 1. die Wiederkunft Christi abwarten und die Beschleunigung der Zeit unter den Vorstößen des Antichrist miterleben - unter Vorstößen, die umso heftiger ausfallen werden, als dieser den Kampf gegen Michael und seine Engel im Himmel verloren hat und nur über ganz wenig Zeit verfügt ${ }^{15}$ (dreieinhalb Jahre) ${ }^{16}$, um, einmal auf Erden losgelassen, seinem Zorn bis zur Parusie die Zügel schießen zu lassen;

10 2. über eine theologisch-politische Revolution gewaltsam eingreifen (wie die böhmischen Taboriten, die Münsteraner Wiedertäufer oder auch Thomas Müntzer und die Bauernkriege), um durch Herbeiführung des Milleniums die Endzeit zu beschleunigen und voranzutreiben, oder in ein anderes innerweltliches Territorium 
auswandern, das der verdorbenen alten Welt, dem Sitz des Antichrist, entrückt ist und als vorparadiesisch gilt (z. B. die von den berühmten Thesen Jung-Stillings angeregte, am Ende des 18. Jahrhunderts einsetzende Auswanderung nach Osteuropa und Südrussland angesichts der erwarteten Heraufkunft des Antichrist und der Aufklärung aus dem Westen, eine Erwartung, die das Schauspiel der Französischen Revolution hinreichend zu belegen schien). ${ }^{17}$

Der in die Niederlande ausgewanderte Pierre Jurieu, seit der Rücknahme des Edikts von Nantes ein entschiedener Gegner der antiprotestantischen Politik Ludwigs des XIV., gibt in seiner Schrift L'accomplissement des prophéties (1686) ein anderes berühmtes Beispiel für politischen Millenarismus. ${ }^{18} \mathrm{Ihm}$ zufolge verkörpert die durch den Papismus vertretene Römische Kirche die Periode des Antichrist und macht eben dadurch deutlich, dass das Ende der Welt nahe ist (der Prophezeiung zufolge in »dreieinhalb Jahren«, also im Jahr 1785). ${ }^{19}$ Diesen literarischen Prophetismus setzten die Kamisarden der Dauphiné und der Cévennen in einen Millenarismus um, der sich der Gefolgsleute der Monarchie ebenso gewaltsam annahm wie er den Zeichen seiner göttlichen Gnadenwahl vertraute. ${ }^{20}$ Auch hier konnte der millenaristische Kommentar der Geheimen Offenbarung des Johannes - in der Tradition des Justinus ${ }^{21}$, Papias ${ }^{22}$ und Sankt Irenäus ${ }^{23}$ - zu Formen gewaltsamen Aufruhrs bei Minderheiten führen, die sich von einer dem Antichrist gleichenden irdischen Macht unterdrückt fühlten.

12 Dieses millenaristische Warten auf "die Beschleunigung der Endzeit» oder die »Abkürzung der Zeiten« blieb lange bestehen, büßte seine ursprünglich apokalyptische Substanz aber nach und nach ein: Der beträchtliche Wandel in den Kenntnissen und Leistungen der Naturwissenschaften (seit dem 17. Jahrhundert) wird in Verbindung mit fortschrittlichen Idealen bezüglich einer gerechteren Neuorganisation der Gesellschaft dieses metaphorische Repertoire auf der Ebene politischer Ideologien und Versprechungen nämlich radikal verzeitlichen. Jurieus antithetisches Vokabular "Millenaristen« versus "Antimillenaristen ${ }^{24}$ - findet sich bald in der revolutionären Rhetorik aufgefrischt wieder. ${ }^{25}$

So spinnt Ferdinand Lassalle 1859 in seinem Franz von Sickingen ${ }^{26}$ die Metapher weiter, verleiht ihr nunmehr jedoch einen neuen, sozialistischen Gehalt (und eine Analogie zur Geburtshilfe). Lassalle gibt zu verstehen, dass der innerweltliche historische Prozess vermittels revolutionärer Gewalt - die für die historische Heraufkunft des Fortschritts dasselbe sein soll wie der Kaiserschnitt für die Gebärende - zwar beschleunigt, in seinem von unwandelbaren Gesetzen des historischen Fortschritts vorgeschriebenen Ablauf jedoch niemals umgelenkt werden könne; diese Möglichkeit historischer Beschleunigung stelle sogar die einzige Manövriermarge dar, über die die Menschen in Bezug auf diese Gesetze verfügten. Besagter "Kaiserschnitt« ist in diesem Rahmen letztlich nichts anderes als der theoretische Ort revolutionärer Gewalt, angesichts der unhintergehbaren Zwänge der Geschichte das einzige Attribut menschlicher Freiheit. Denn zwar kann die Revolution beschleunigt, keineswegs aber unbeschränkt aufgehalten werden: Wenn ihre Stunde geschlagen hat, muss sie eintreten. 


\section{Das Aufkommen des Begriffs "Geschichte" und die beiden Revolutionen}

\section{Der Begriff Geschichte}

14 die "Beschleunigung der Geschichte« zum Thema wird - zu einem Thema, das am Ende des 18. Jahrhunderts den früheren Diskursen über die »Beschleunigung des Endes der Zeiten « nach und nach den Rang abläuft. Bevor die umgreifende Kategorie einer linear ausgerichteten, kumulativ verlaufenden, am Zeitpfeil sich ausrichtenden "Geschichte« sich im mentalen Raum der Europäer einnistet, lässt sich tatsächlich nicht von einem Gefühl der »Beschleunigung der Geschichte« sprechen. ${ }^{27}$ zugrunde, dann bedeutet, was dort "Zukunft" heißt, noch absolut nicht das, was wir heute darunter verstehen. Zunächst einmal gibt es den Begriff nicht in der Einzahl: Was es gibt, sind zweierlei Dinge, die erst am Ende des 18 . Jahrhunderts konvergieren ${ }^{28}: 1$ ) die Zukunft im Sinne des Adventus Domini (»Zukunft des Herrn«) ${ }^{29}$, das heißt der Wiederkehr Christi auf Erden am Ende der Zeiten (also die zweite Parusie); 2) die Vorstellung »künftiger Dinge«, die jenseits der bloßen Gegenwart eintreffen sollen, also der Plural futura oder »Zukünftiges«. ${ }^{30}$

Neu ist im 18. Jahrhundert das Aufkommen des mentalen Raums der "Zukunft« als solcher (unsere noch nicht gänzlich säkularisierte, aber auf den Begriff einer linear verlaufenden Geschichte schlechthin bezogene Zukunft) - ein Aufkommen, das mit der semantischen Transformation der "Zukunft des Herrn« in »Zukunft des Menschen« einhergeht. Der Singular Zukunft und der Plural futura fusionieren also:

1. zunächst einmal über eine allmähliche Säkularisierung des Adventus-Motivs. Die Frage des Sinns des menschlichen Treibens auf Erden fällt nunmehr in die Kompetenz der fortschrittlichen Geschichtsphilosophien (die "Geschichtsphilosophie " tritt erst etwa im zweiten Drittel des 18. Jahrhunderts als eigene Disziplin auf - als erster spricht 1765 Voltaire von philosophie de l'histoire); wir wohnen gewissermaßen einer »Verlagerung der Hoffnung ( (um die glückliche Formulierung Halévys aufzugreifen) ${ }^{31}$ oder auch einer "Verzeitlichung « der herkömmlichen messianischen Hoffnungen bei. ${ }^{32}$ Des Problems der Hoffnung nimmt sich nunmehr das Schema des »Fortschritts« an. In seinem berühmten Text über Die Erziehung des Menschengeschlechts (§ 80) spricht Lessing es 1777 aus. Es hilft nichts zu wollen, dass die Geschichte und das Ende der Zeiten sich nicht beschleunigen mögen, es gilt, sich mit der Geduld des Fortschritts zu versehen, der sich langsam ausbreitet, und sich darauf einzustellen, die Verwirklichung des Versprechens selbst nicht zu erleben. Die Säkularisierung ist nicht vollständig beileibe nicht -, aber die herkömmliche apokalyptische und chiliastische Literatur über die Beschleunigung des Endes der Zeiten lassen wir allmählich hinter uns. Auf die eschatologische Ungeduld des Schwärmers folgt nach und nach die fortschrittliche Gestalt des Geschichtsphilosophen, des unermüdlichen Entzifferers des Sinns der Ereignisse hienieden;

18 2. sodann über die Singularisierung von futura in futurum. Diese Umformung wird von dem Aufkommen des Begriffs Geschichte begleitet (nicht mehr »der Geschichten« im 
Plural, sondern auch hier »der Geschichte« schlechthin, die nunmehr in einer bestimmten Richtung verläuft und irreversibel ist). ${ }^{33}$

»Zukunft«, "Fortschritt«, "Geschichte« sind allesamt »kollektive Singulare«, die am Ende des 18. Jahrhunderts auftauchen und ohne die selbst die Vorstellung von einer »Beschleunigung der Geschichte« als solcher einfach nicht denkbar ist.

\section{Eine allgemeine Epochendiagnose: Französische Revolution und industrielle Revolution}

Das am Ende des 18. Jahrhunderts denkbar gewordene Motiv der "Beschleunigung der Geschichte« verwandelt sich zu Beginn des 19.Jahrhunderts in eine generelle Epochendiagnose. Die Entwicklung der Semantik und der Empfindungen verzeichnet somit die Auswirkungen einer doppelten, dauerhaften Erschütterung: die der Französischen Revolution im politischen und symbolischen Bereich; die der industriellen und technischen Revolution im Feld der sozialen Interaktionen.

21 Dazu gibt es eine Unzahl von Zeugnissen. Besonders aufschlussreich berichtet zu den Folgen der Französischen Revolution Karl-Viktor von Bonstetten (1745-1832):

"Ich habe einige Erinnerungen aus einem sehr vielgestaltigen Leben dargestellt; ich habe die Sitten der Nationen nachgezeichnet, die ich kennenlernte; aber die Mehrzahl dieser Bilder gehören nunmehr einer anderen Welt an, einer altertümlichen Welt, einer Epoche jenseits der großen historischen Schranke, die den Namen Revolution trägt. Fast alle diese Bilder sind verschwunden und haben nur Bruchstücke hinterlassen, die uns an etwas erinnern, das nicht mehr ist. Wir sehen, dass die Alpen zwei Völker trennen, die einander keineswegs gleichen. So steht es auch mit dem großen Gebirgszug zwischen zwei Zeitaltern; er trennt Menschen, die sich derart voneinander unterscheiden, dass diejenigen, die wie ich in beiden Epochen gelebt habe, sich wundern, dieselben Menschen zu sein. « $^{34}$

Bonstetten führt hier mehrere Leitmotive der Epoche zusammen: das Gefühl einer unerhörten Beschleunigung der politischen und sozialen Zeit; die Abtrennung der Gegenwart von der Vergangenheit, die uns damit ebenso fremd wird wie wir uns selbst; und schließlich die räumliche Metaphorisierung der Zeit mit jenem "Gebirgszug« der Französischen Revolution, die zwei getrennte, nunmehr in sich geschlossene Räume der Wahrnehmung und Selbstwahrnehmung schafft.

Als Beispiel ließe sich auch Chateaubriand zitieren, der, als »Historiker« allzu oft vergessen, in seinen Historischen Studien von 1831 erläutert, wie die zeitgenössische Beschleunigung der Geschichte ihn in seiner Arbeit als Historiker tangiert (er schreibt gerade über Henri IV. und die Religionskriege in Frankreich):

"Die lebendige Geschichte hat die einstmals so berühmten Vorfälle der toten Geschichte kleiner werden lassen. Was gilt der Tag der Barrikaden, was selbst die Bartholomäusnacht angesichts jener großen Erhebungen vom 7. Oktober 1789, vom 10. August 1792, angesichts der Massaker vom 2., 3. und 4. September desselben Jahres, der Ermordung Ludwigs des XVI., seiner Schwester und seiner Frau, was schließlich angesichts der ganzen Schreckensherrschaft? Und während ich mich jener Barrikaden annahm, die einen König aus Paris vertrieben [12. Mai 1588], brachten andere Barrikaden [die des Juli 1830 in Paris] innerhalb weniger Stunden drei Generationen von Königen zum Verschwinden. Die Geschichte wartet nicht mehr auf den Historiker: Sie zieht einen Strich; sie rafft eine Welt hinweg. ${ }^{35}$

Die Beschleunigung der Zeit führt daher geradezu zur Unfähigkeit, Geschichte zu schreiben (zumal zeitgenössische Geschichte, Gegenwartsgeschichte). Wie die 
Zeitgenossen zugestehen, ist dies völlig paradox, da das Bedürfnis nach Orientierung gerade in dieser Krisenzeit am intensivsten spürbar wird.

Aber das Wesentliche, das, worum es grundsätzlich geht, ist der prinzipielle und symbolische Wandel der politischen Ordnung.

»Namenlose Massen bewegen sich wie die Volksbewegungen des Mittelalters, ohne zu wissen weshalb, ausgehungerte Herden, die keinen Hirten noch anerkennen, die von der Ebene ins Gebirge und vom Gebirge in die Ebene rennen, die die Erfahrung der in Wind und Wetter abgehärteten Hirten mißachten. Im Leben der Gemeinwesen ist alles im Wandel begriffen. Religion und Moral werden nicht mehr anerkannt, oder jeder interpretiert sie auf seine Art. In weniger wichtigen Fragen die gleiche Ohnmacht der Überzeugung und des Daseins: ein Ruhmestitel leuchtet kaum eine Stunde: ein Buch altert in einem Tag; Schriftsteller nehmen sich das Leben, um die Aufmerksamkeit auf sich zu lenken; weitere Eitelkeit: man hört nicht auf ihre letzten Seufzer. [...] An die Stelle der Invasion der Barbaren ist die Invasion der Ideen getreten; die gegenwärtige aufgelöste Zivilisation verliert sich selbst; die Vase, die sie enthält, hat die Flüssigkeit noch nicht in eine andere Vase gegossen; die Vase ist zerbrochen. [...]Wenn die Dampfmaschine vervollkommnet sein wird, wenn durch sie, durch den Telegraphen Entfernungen unbedeutend geworden sind, werden nicht nur die Waren reisen, sondern auch die Ideen auf Reisen gehen! Wenn die Schranken des Fiskus und des Handels zwischen den verschiedenen Staaten niedergelegt sein werden, wie sie es zwischen den Provinzen ein und desselben Staates schon sind, wenn die verschiedenen Länder in täglicher Beziehung nach der Einheit ihrer Völker streben werden, wie wollt ihr dann die alte Form der Trennung wieder zum Leben erwecken? «36

Die Zerrüttung der »alten Form der Trennung« auf allen Ebenen der Gesellschaft: eben darum geht es.

Somit intensivieren die vom technisch-industriellen Wandel des frühen 19. Jahrhunderts hervorgerufenen materiellen Beschleunigungen allenfalls den durch die Auflösung der politischen, sozialen und religiösen Erfahrungsrahmen des Ancien Régime ausgelösten großen Transformationsprozess. Wenn »die alte Welt endet ${ }^{37}$, so deswegen, weil wir mit dem Jahr 1789 in die wortklauberische Ära der großen Prinzipien und Ideologien eingetreten sind, in der Nationen oder Massen die Legitimität und Erfahrung der Tradition verdrängen. Das Thema der Beschleunigung der Geschichte, für manche ein Symbol der Besorgnis, für andere ein Symbol grenzenloser Hoffnung, stellt für das Bewusstsein beider Gruppen den Übergang von einem theologisch-politischen Zeitalter zu einem anderen dar: Macht und Autorität der »in Wind und Wetter abgehärteten Hirten« haben sich überlebt, während das ungewiss bleibende Gleichgewicht der modernen demokratischen Ordnung sich gerade erst einstellt. Während die Ungewissheit über die Zukunft steigt, ist Epochendiagnose eher eine Beschwörungsformel als ein analytischer Begriff, eher recht unbestimmtgefühlsmäßiger Natur als ein Urteil. Kurz, die Thematik der Beschleunigung der Geschichte entspricht anfänglich einem Versuch aktiver Aneignung dessen, was die Subjekte in der Entwicklung ihrer Gesellschaft absolut in Mitleidenschaft zieht und lähmt ${ }^{38}$; sie verrät ein nicht zu unterdrückendes Bedürfnis nach Orientierung und geht letzten Endes aus der Kantschen Thematik des historischen Symbols im Streit der Fakultäten hervor. ${ }^{39}$

Was nun die Gesamtheit dieser Diskurse thematisiert, ließe sich als fortschreitende Entnaturalisierung der herkömmlichen Zeiterfahrung bezeichnen, die früher viel stärker an natürliche Lebenszwänge gebunden war (Tag, Nacht, langsame Fortbewegung im Raum durch tierische Zugkräfte usw.). Die durch die Mechanisierung und 
Industrialisierung motorischer Kräfte hervorgerufene neue Zeiterfahrung markiert das baldige Ende eines mehrere hundert Jahre währenden Zeitalters: des hippomobilen Zeitalters, von dessen Zwängen die Menschen sich jahrhundertelang nur teilweise $\mathrm{zu}$ befreien wussten (durch Maximierung ein und derselben Fortbewegungsart - Pferd und Gespann - durch intensivere Nutzung der Post, Verbesserung der Straßen, Verringerung der Kosten usw.). ${ }^{40}$ Und ebendiese Erfahrung vom Ende des Zeitalters, in dem das Pferd die schnellstmögliche Bewegung im Raum darstellte ${ }^{41}$, verstärkte das Gefühl, dass sich ein neues politisches und kulturelles Zeitalter auftat: nicht mehr Trennung der Ordnungen und Stände, sondern Fusion und Agitation der Massen; nicht mehr der Absolutismus des Ancien Régime, sondern die Egalisierung der Lebensbedingungen und die demokratische Dynamik mittels einer stets labilen Spannung zwischen Freiheit und Gleichheit, zwischen der Quelle der Souveränität und ihrer institutionellen Formgebung durch die repräsentrative Regierung.

Dieses Gefühl materieller Beschleunigung wandelt sich in den Zeit-Diskursen in Form verschiedener Unterthematiken ab.

a) Die Beobachter haben zunächst den Eindruck, dass der Raum schrumpft wie niemals zuvor. Der Brockhaus stellt dies $1838^{42}$ fest, während Heinrich Heine in einem Text, den er 1843 in Lutetia aufnahm, wörtlich schreibt:

»Aber die Zeit rollt rasch vorwärts, unaufhaltsam, auf rauchenden Dampfwagen, und die abgenutzten Helden der Vergangenheit, die alten Stelzfüße abgeschlossener Nationalität, die Invaliden und Incunabeln, werden wir bald aus den Augen verlieren. [...] Wir merken [...], daß unsre ganze Existenz in neue Gleise fortgerissen, fortgeschleudert wird, daß neue Verhältnisse, Freuden und Drangsale uns erwarten, und das Unbekannte übt seinen schauerlichen Reiz, verlockend und zugleich beängstigend. So muß unsern Vätern zu Muth gewesen sein, als Amerika entdeckt wurde, als die Erfindung des Pulvers sich durch ihre ersten Schüsse ankündigte, als die Buchdruckerei die ersten Aushängebogen des göttlichen Wortes in die Welt schickte. Die Eisenbahnen sind wieder ein solches providencielles Ereigniß, das der Menschheit einen neuen Umschwung giebt, das die Farbe und Gestalt des Lebens verändert; es beginnt ein neuer Abschnitt in der Weltgeschichte, und unsre Generation darf sich rühmen, daß sie dabei gewesen. Welche Veränderungen müssen jetzt eintreten in unsrer Anschauungsweise und in unsern Vorstellungen! Sogar die Elementarbegriffe von Zeit und Raum sind schwankend geworden. Durch die Eisenbahnen wird der Raum getödtet, und es bleibt uns nur noch die Zeit übrig. Hätten wir nur Geld genug, um auch letztere anständig zu tödten! In vierthalb Stunden reist man jetzt nach Orléans, in ebenso viel Stunden nach Rouen. Was wird das erst geben, wenn die Linien nach Belgien und Deutschland ausgeführt und mit den dortigen Bahnen verbunden sein werden! Mir ist, als kämen die Berge und Wälder aller Länder auf Paris angerückt. Ich rieche schon den Duft der deutschen Linden; vor meiner Thüre brandet die Nordsee.« ${ }^{43}$

b) Ferner entwickelt sich das Gefühl, dass die neuen Verkehrsmittel wie Eisenbahn und Dampfschiff - da sie faktisch die Klassenspaltung aufheben, indem sie alle Welt mit derselben Geschwindigkeit und $\mathrm{zu}$ immer niedrigeren Kosten befördern - die demokratische und egalitäre Tendenz der modernen politischen Ordnung beschleunigen. Nicht nur in der theologisch-politischen und in der sozialen Ordnung, sondern auch in der materiellen und alltäglichen ist die Gleichheit der Lebensbedingungen im Vormarsch. Bald wird es aus sein mit dem Paris, das LouisSébastien Mercier 1771 beschrieb und dessen Straßen im Wesentlichen auf Grund der ostentativen Eitelkeit der Großen und Reichen, die "vergessen [hatten], daß [sie] Beine zum Gehen [besaßen] «, mit Sänften und Privatkarossen verstopft waren. ${ }^{44}$ 

sozialen Klassen aus und verwandelt sie in eine sich immer schneller fortbewegende, homogene Masse, deren Bahn für jeden dieselbe ist und die bei Bedarf durch oder über einstige natürliche Hindernisse wie Gebirge, Schluchten, Flüsse, Täler usw. führt. In der Kunst - wie wenig später auch in der Werbung - treten neue ikonographische Register in Erscheinung: An die Stelle des romantisch Erhabenen, wie es das machtvolle Schauspiel der Natur bietet, treten gerade Linien, auf denen sich die Geschwindigkeit im Raum der modernen Künstler über Reliefs hinweg hin zu dem einzigen Horizont entfaltet, der ihrem himmelstürmerischen Drang noch Widerstand bietet - der Sonne.

c) Die festgestellte Beschleunigung berührt gleichfalls die soziale Arbeitsteilung und den rascheren Rhythmus der Produktionsprozesse. Die der Technik geschuldeten Produktivitätsgewinne erlauben ein Absenken der in den Produktionskosten eingeschlossenen Lohnkosten; immer mehr Menschen finden sich durch Maschinen ersetzt, und schon entstehen neben der Sorge um die Arbeitslosigkeit andere Ängste wie etwa die, die Chateaubriand in seinen Erinnerungen so formuliert: "Was soll man mit dem beschäftigungslosen Menschengeschlecht anfangen? « ${ }^{45}$

traditionellen Produktionsprozessen abgezwungenen Freizeit machen werden; und wer sich - wie Chateaubriand - auf den Standpunkt eines katholischen Denkens stellt, das die Erbsünde und das Ringen mit der Materie zu Bedingungen der Möglichkeit menschlicher Würde und Freiheit erhebt, der kann diese gewonnene Zeit nur als Quelle künftiger Laster der arbeitenden Klassen verstehen. Für den, dem vor dem Zeitalter der Massen graut, verstärken technischer wie industrieller Fortschritt und demokratische Gleichmacherei sich gegenseitig und erscheinen als Träger aller künftigen soziopolitischen Störungen. Ein und dieselbe Erfahrung materieller Beschleunigung kann hier jedoch zu unterschiedlichen Reaktionen führen (zur antidemokratischen Besorgnis Chateaubriands wie zur fortschrittlichen Ironie Heines $\left.{ }^{46}\right)$, sodass die von einem gemeinsamen Rahmen von Phänomenen ausgehende Epochendiagnose heterogener Beobachter unter der Einwirkung soziopolitischer, religiöser und kultureller Voreinstellungen in unterschiedliche Richtungen gehen kann. Einer noch zu begründenden historischen Soziologie der materiellen und sozialen Beschleunigung der Moderne fiele die Aufgabe zu, diese oder jene Voreinstellung mit diesem oder jenem Typus von Zeitdiagnose empirisch zu korrelieren.

d) Schließlich hat man das Gefühl, dass die von den modernen Menschen erfahrene soziale Beschleunigung mit ihrer Egalisierung der Lebensbedingungen im symbolischen und politischen Bereich das einst strikt natürliche System der Bedürfnisse umgreifend verändert. Die bloße Zeit des Ausruhens, d.h. des Regenerierens der Arbeitskraft zu Zwecken des Überlebens, wird durch eine an sich freie Zeit ersetzt, die neue Wünsche hervorruft, die sich dem Bewusstsein nach und nach als neue Bedürfnisse darstellen. Nun entsteht das Bedürfnis der Intensivierung der Bedürfnisse und Vergnügungen als neue Lebensweise; nun entstehen das System der Mode und das Reich des Ephemeren, die Baudelaire zum Ausgangspunkt seiner Definition des eigentlichen Wesens der Moderne erhebt. ${ }^{47}$ Natürlich betrifft dieses System anfangs nur eine kulturelle und soziale Elite; da jedoch der Anteil der Freizeit in Abhängigkeit von der zunehmenden Produktivität im wirtschaftlichen Bereich auch unter den Massen wächst, entsteht hier ein paradigmatisches Ethos, das immer mehr um sich greift. Das Aufkommen der Thematik der Langeweile - von ihrer trivialen sozialen Dimension bis hin zur 
metaphysischen - ist von diesen unterschiedlichen Erfahrungen sozialer Beschleunigung nicht zu trennen. ${ }^{48}$

Zwei Reihen von Diagnosen und Argumenten sind - so sehr sie auch miteinander einhergehen und sich wechselseitig verstärken - bei ihrer Analyse sorgfältig auseinanderzuhalten und zueinander ins Verhältnis zu setzen: die eine, die sich auf die Französische Revolution bezieht und daher mit dem Wandel der modernen soziopolitischen Ordnung zusammenhängt; und die zweite, die sich auf die industrielle Revolution bezieht und die Transformationen des materiellen und ökonomischen Rahmens des modernen Lebens betrifft. Das Gefühl der Beschleunigung geht jedoch nicht unbedingt mit dem Gefühl radikaler Neuartigkeit einher. Die Französische Revolution konnte in diesem Sinn manchmal als eine kurze Periode wahrgenommen werden, während der alle seit der Antike theoretisch gefassten politischen Formen aktualisiert und beschleunigt wiederkehrten, ohne doch eine qualitative Innovation $\mathrm{zu}$ beinhalten. Die Vorstellung einer absoluten Neuartigkeit historischer Zeiten geht daher nicht zwangsläufig mit der Feststellung einer Form sozialer Beschleunigung einher: Das Alte kann sich auch schlicht und einfach im Zeitraffer wiederholen.

\section{Die Beschleunigung der Geschichte heute: von der Globalisierung zum Ideal der Entschleunigung}

Das Thema »Beschleunigung der Geschichte» deckt sich heute mit zahlreichen heterogenen Analysen, die wir in einige große Gruppen zu gliedern versuchen werden.

\section{Die Globalisierung}

Die Thematik »Beschleunigung der Geschichte« wird heute immer häufiger mit der der Globalisierung in Verbindung gebracht. Damit wird zunächst einmal der Zustand einer durch die Verallgemeinerung der Informatik und die Verbreitung des Internets, durch Mobiltelefone und Satellitenfernsehen mit Netzwerken überzogenen zeitgenössischen Welt beschrieben; ferner werden dabei die durch Senkung der Transportkosten ermöglichten Betriebsverlagerungen ins Auge gefasst, die es erlauben, aus der Differenz der Lohnkosten zwischen den verschiedenen Wirtschaftsräumen Gewinn zu erzielen; schließlich unternimmt man, auf diese Weise die zeitgenössische Finanzialisierung der Wirtschaften $\mathrm{zu}$ beschreiben, die bewirkt, dass - um eine berühmte Formel Karls V. abzuwandeln - »die Sonne über dem Reich der Finanzen niemals untergeht«: Die Zeit der Interaktion in einer finanzialisierten Wirtschaft wäre damit heute die zur Durchführung globaler Transaktionen erforderlich Mindestzeit, also nahezu die Zeit, die das Licht braucht - die Echtzeit. ${ }^{49}$

\section{Die Intensivierung sozialer und politischer Interaktionsbeziehungen}

Die Thematik »Beschleunigung der Geschichte» wird oft mit der der Beschleunigung des Rhythmus sozialer Interaktionen und deren Auswirkungen auf das individuelle Bewusstsein verwechselt. Daher wird der von Simmel an der Schwelle zum 20. Jahrhundert eingeführte Unterschied zwischen »Tempo des Lebens« und »Zeit des Lebens ${ }^{50}$, d. h. zwischen erlebter Zeit (der Stelle aller Empfindungen) und kalendarisch messbarer Zeit (die sich ihrerseits streng genommen nicht beschleunigen kann), kurz 
zwischen subjektiver und objektiver Zeit, in den Untersuchungen verwischt. Was dann mit dieser Thematik bezeichnet wird, ist die Tatsache, dass auf die Individuen der modernen (industrialisierten, verstädterten, massenbasierten, kurz funktionell differenzierten) Gesellschaften innerhalb ein und derselben kalendarisch messbaren Zeiteinheit im Vergleich zu ihren Vorfahren immer mehr und immer kompaktere Ereignisse und soziale Beziehungen einwirken, und zwar so sehr, dass sie unter dem Ansturm dieser von allen Seiten auf sie einströmenden sozialen Wirklichkeit schließlich das Gefühl haben, dass ihre psychische Ökonomie von der Bewältigung der sie überflutenden, unendlichen Vielfalt überfordert ist. Das Thema der "Beschleunigung der Geschichte" (oder auch der "Beschleunigung der Beschleunigung « seit dem Aufkommen der modernen Kommunikations- und Informationstechnologien, das dem vom elektrischen Telegraphen um 1850 ausgelösten Wandel ${ }^{51}$ eine exponentielle Dimension verleiht), ist nunmehr semantischer Index einer psychischen Bruchstelle, der der moderne Mensch unfehlbar früher oder später zum Opfer fallen soll. An die Stelle einer Vision vom zwangsläufig eintretenden Fortschritt der Geschichte ist seit Ende des 19.Jahrhunderts das »Zeitalter der Nervosität « ${ }^{52}$ getreten, und die gegenwärtige Epoche transponiert diese wohlbekannte Szene gewissermaßen nur ins Hightech-Zeitalter der Netzwerkanbindung.

Zweierlei allerdings ist neu. Zum einen ist die kürzlich erfolgte Erhebung von Flexibilität und rastloser Arbeit zur Norm ein untrügliches Zeichen dafür, dass die soziale Wertigkeit des einstigen adligen Müßiggangs (den der bürgerliche Rentier im 19. Jahrhundert nachäffte) umgekehrt wurde: Rastlosigkeit wurde zum äußeren Kennzeichen des sozialen und symbolischen Kapitals eines Individuums, und ostentativer, sich selbst genügender Aktionismus ist seither nicht mehr nur ein Zeichen sozialer Distinktion, sondern eine ökonomische Statusideologie. ${ }^{53}$ Zum anderen hat das Reich des in Echtzeit über den ganzen Planeten hin ausgestrahlten Events die politische Gesamtlage gegenüber dem »Zeitalter der Nervosität« am Ende des 19. Jahrhunderts beträchtlich modifiziert. Die Wende zur Mediatisierung, von der die örtliche wie die globale Politik in den letzten beiden Jahrzehnten erfasst wurden, hat eine bislang in der Geschichte nie dagewesene, fast vollständige Konvergenz zwischen einem Ereignis und seiner der internationalen Öffentlichkeit zugänglichen Fernsehübertragung herbeigeführt. Angesichts eines wie auch immer gearteten politischen Geschehens, das unmittelbar von den Fernsehstationen - oder jetzt auch von Bloggern und Podcastern usw. - aufgegriffen wird, schrumpft die Zeit, die sich die unterschiedlichen politischen Akteure für ihre Reaktion nehmen können, immer mehr dahin; manche gehen so weit zu behaupten, dass dringende politische Lösungen nunmehr in Echtzeit, das heißt mit der Geschwindigkeit der Bildübertragung, gefunden werden müssen. Darauf hat der amerikanische Generalstab 2003 während des zweiten Irak-Feldzugs in der Weise reagiert, dass er in die angreifenden Truppen Kriegsreporter »einbettete«: Die sich vor unseren Augen in Echtzeit ereignende Geschichte war von der sich als vollständige Transparenz tarnenden Zensur nicht mehr zu trennen.

Allem Anschein nach ist damit eine neue Phase eingetreten: die des politischen Zeitdrucks und des damit einhergehenden Begriffs des "politischen Risikos «. ${ }^{54}$ Wenn für manche das Zeitalter der Arkana der Macht, in denen die Regierenden sich eine den Medien unzugängliche und geheime Zone einrichten konnten, heute definitiv zu Ende ist, darf man sich fragen, ob die Bedingung der Möglichkeit aller Politik und 
Handlungsfreiheit nicht immer noch das "Geheime« bleibt, mit einem Wort: ein Mindestmaß an Reserviertheit des Staats, der Regierenden und der zentralen Entscheidungsapparate. ${ }^{55}$ Anders gesagt: Die Thematik "politischer Zeitdruck " geht allzu rasch an der heute in Blüte stehenden Soziologie der politischen "Entscheidung" vorbei.

\section{Die Trägheit der technischen und industriellen Systeme als innerweltliche Eschatologie} auch unter dem Gesichtspunkt ihrer langfristigen Wirkungen ins Auge gefasst. Die Trägheit der technischen, industriellen Systeme und ihrer langfristigen Auswirkungen erscheint so als eine der Hauptquellen zeitgenössischer Ängste. Uns schwant, dass das Los schon gefallen ist, dass die Tage unseres Daseins hienieden und die des Planeten vielleicht schon gezählt sind, und wir verlängern täglich die Liste der unter den vereinten Auswirkungen der Wissenschaft und ihrer täglichen Anwendungen, der industriellen Systeme und der Konsumgewohnheiten in den entwickelten Ländern möglich gewordenen ökologischen Katastrophen. Da eine solche Katastrophe nie allein eintritt, stehen uns die menschlichen, geopolitischen usw. Katastrophen vor Augen, die dem unmittelbar folgen würden. mentale Raum einer neuen Eschatologie herausgebildet - nicht mehr der chiliastischen Eschatologie von einst, sondern einer Art paradoxer innerweltlicher Eschatologie: Wir wissen, dass das Dasein der Menschheit auf Erden an sein Ende gelangen kann, wissen aber aufgrund der zu großen Komplexität der fraglichen Systeme und klimatischen Variablen noch nicht, innerhalb welcher Frist dieses Ende kommen kann. Daher polarisieren sich die Debatten: Auf die "Heuristik der Angst« (Hans Jonas) reagieren voluntaristische Diagnosen, denen zufolge die Moderne wie stets in sich selbst (im Instrument ihres möglichen Unglücks - der Wissenschaft - und in ihrer privilegierten, da der Selbstreflexion und Selbstkorrektur fähigen politischen Form - der Demokratie) die zur langfristigen Sanierung einer verfahrenen Situation notwendigen Ressourcen finden werde. ${ }^{56}$

Unsere heutige »Weltrisikogesellschaft ${ }^{57}$ verweist damit auf eines der größten Paradoxe unserer Zeit: Wir können nicht mehr wie einst der überwältigenden Allmacht der Natur oder der Götter die Verantwortung für Naturkatastrophen zuschieben, aber zugleich können wir nicht diesen oder jenen Politiker herausgreifen, um ihn für Entscheidungen verantwortlich zu machen, die er getroffen hat bzw. nicht rechtzeitig treffen wollte oder konnte, um zu verhindern, dass sich dieses oder jenes weltweite Gefahrenpotential verschärft. Angesichts der kurzfristigen Trägheit der ökonomischen Systeme einerseits und der geopolitischen Kräfteverhältnisse andererseits legt die innerweltliche ökologische Eschatologie gewissermaßen den Finger auf das, was in Wahrheit die Quadratur des Kreises der Temporalitäten heutigen politischen Handelns darstellt: d. h. auf die unmögliche Harmonisierung der Langfristigkeit der von der globalen technisch-industriellen Sphäre initiierten ökologischen Auswirkungen und der kurzen oder mittleren Frist demokratisch legitimierten politischen Handelns.

Die humanisierte / technisierte Natur hat somit strukturell den einst vom Zufall der Naturkatastrophen (Erdbeben, Vulkanausbrüchen usw.) besetzten Platz übernommen; 
da in den Augen der Staatsbürger die zeitlichen Dimensionen politischen Handelns nicht mehr auf der Höhe der eingegangenen Risiken sind, wohnen wir einer zunehmenden Entlegitimierung der demokratischen politischen Systeme bei. Die von Francis Bacon datierende moderne und fortschrittliche Gleichsetzung von Beherrschung der Natur und Beherrschung des kollektiven Geschicks einer Gesellschaft oder gar der Menschheit scheint heute definitiv desavouiert:

"Es sieht manchmal so aus, als ob die Gesellschaften ihr Schicksal um so weniger bestimmen, je mehr sie, mit dem Mittel der Technik, eine verstärkte Herrschaft über die natürliche Umwelt gewonnen haben. ${ }^{58}$

\section{Die Entsynchronisierung der politischen Zeitdimensionen}

Der zuletzt erwähnte Punkt führt uns auf ein von der gegenwärtigen sozialen Beschleunigung ausgehendes allgemeines Problem. Seit über zwei Jahrhunderten bestand der große Ehrgeiz der Moderne sicherlich darin, einen Zivilisationstypus zu formen, in dem das Ideal individueller und kollektiver Autonomie endlich dauerhaft verwirklicht werden könnte. Der Staat des Ancien Régime und die damaligen militärischen Erfordernisse hatten bereits eine gewisse administrative und territoriale Zentralisierung und Regulierung sowie eine technische Innovation in Gang gebracht. Darauf aufbauend wurden die Strukturen demokratischer Beschlussfassung im Rahmen dessen, was Ulrich Beck die »erste Moderne" nennt, als Beschleunigungsfaktoren des Emanzipationsprojekts konzipiert. Das damalige historische Bewusstsein konnte sich daher eine vollkommene und dauerhafte Übereinstimmung von politischen Formen und ökonomisch-technischen Prozessen erträumen, die der Produktion von Reichtum (und über Verteilungsmechanismen herzustellender Autonomie) innerhalb jeder bürgerlichen Gesellschaft zugrunde lägen. Was wir heute - unter dem Eindruck der nicht nur von Generation zu Generation, sondern selbst innerhalb jeder Generation wahrnehmbaren sozialen Beschleunigung - erleben, ist die je nach Land und jeweiliger Lage mehr oder weniger starke "Entsynchronisierung « zwischen den regulierenden Rahmenbedingungen dieser oder jener politischen Einheit und dem galoppierenden Wandel der jeweiligen wirtschaftlichen und technischen Strukturen. Aus dieser "Entsynchronisierung" schließen manche Autoren (z. B. William F. Scheuerman) $)^{59}$ sogar auf die Notwendigkeit, selbst den Kern der modernen Demokratietheorien, nämlich das Prinzip der Gewaltenteilung, zu überdenken.

Demnach wäre die repräsentative Regierung des klassischen politischen Verfahrensliberalismus unfähig geworden, hinreichend rasch auf die in unseren Tagen sich immer häufiger einstellenden politischen Notlagen zu reagieren, und zwar eben aufgrund der Zeit, die der parlamentarischen Beratung über Gesetze zu komplexen Problemen zusteht - zu Problemen, über die die Wissenschaften selbst im Übrigen die größte Mühe haben, unabweisbare, konsensfähige Urteile zu fällen. Abgesehen von dem chronischen Rückstand der Legislative gegenüber neuen Phänomenen - zumal wenn sie juristisches Neuland darstellen - werden hauptsächlich zwei negative Auswirkungen festgestellt: Zum einen nutze die Exekutivgewalt Schnellverfahren (so die französische Regierung den Artikel 49 Abs. 3) ${ }^{60}$, die, obgleich verfassungskonform, manchmal missbräuchlich eingesetzt werden, um heikle und unaufschiebbare Probleme beschleunigt zu lösen; zum anderen zeigten Exekutive wie Legislative zunehmend die fatale Tendenz, die demokratische Beratung bzw. Beschlussfassung durch das Parlament zu externalisieren und an andere, demokratisch nicht legitimierte 
Instanzen abzutreten (Expertenkommissionen, Subsysteme der Rechtsprechung, Deregulierung, Privatisierung usw.). Da sie dennoch für den generellen Kurs aller politischen Maßnahmen vor dem Volk verantwortlich bleiben, geraten die Regierungen in eine doppelte Zwickmühle: einerseits, weil sie an demokratisch nicht legitimierte Subsysteme Regulierungsverfahren delegieren, für die sie zwangsläufig vor der Wählerschaft geradestehen müssen; andererseits dann, wenn im Anschluss an außerparlamentarische Beratungen nichtsdestotrotz im Parlament über gesetzliche Maßnahmen abgestimmt wird, weil ihr rechtlicher Gehalt (in bioethischen Fragen zum Beispiel) oft genug über den Common sense von Berufspolitikern hinausgeht.

Dieses aktuelle Phänomen der "Entsynchronisierung" differenzierter Subsysteme in unseren Gesellschaften kann indessen auch andere Seiten zeitigen. Zum Beispiel tendiert der von der Öffentlichkeit auf politische Führer und Apparate ausgeübte Druck dazu, die an Wahlterminen orientierte Kurzfristigkeit politischen Handelns weiter zu verstärken und traditionsorientierte oder sogar mehr oder weniger zweifelhafte Wählergruppen $\mathrm{zu}$ bedienen. So erscheint der gegenüber der »Repräsentationsdemokratie « zunehmende Einfluss der »Meinungsdemokratie« als durch die Medien verstärkter Faktor für die Zuspitzung besagter Entsynchronisierung. ${ }^{61}$ Diese lässt sich darüber hinaus innerhalb ein und desselben administrativen Raums feststellen (vgl. die französischen Banlieue-Unruhen im Herbst 2005). Da gewisse ökonomische, soziale, urbane usw. Situationen eine beträchtliche Trägheit aufweisen, die keinerlei punktuelle politische Aktion aufheben oder spürbar korrigieren kann, erscheinen die demokratisch beauftragten politischen Akteure in den Augen der Öffentlichkeit tatsächlich entweder als unfähig, zynisch und korrupt oder als strukturell ohnmächtig, zumal der mangelnde Weitblick des Staates in der Vergangenheit die aktuellen sozialen Wirren in bestimmten Zonen des nationalen Territoriums nicht voraussah, in denen sich nun auf begrenztem Raum alle Schwierigkeiten der heutigen Welt kristallisieren. Da manche politische Strategien sich heute als gegenüber den in diesen Vierteln real angehäuften Problemen entsynchronisiert erweisen, ist die politische Klasse dauerhaft entlegitimiert, und diese »sensiblen Zonen« des Staatsgebiets erscheinen als wahre "Ghettos auf Zeit«, in denen andere Sitten herrschen und das Gesetz der Republik außer Kraft gesetzt ist. Von diesen innerhalb eines gegebenen Staatsgebiets geltenden unterschiedlichen Temporalitäten (die im Übrigen durch das Versagen der in der Vergangenheit praktizierten Politik erklärbar sind) ist es nur ein Schritt hin zu dem angeblich unassimilierbaren Charakter mancher immigrierter Populationen - ein Schritt, den der zeitgenössische rassistisch angehauchte Populismus natürlich nicht unterlässt.

Die Hauptthese des Soziologen Hartmut Rosa ist unter diesem Gesichtspunkt alles andere als irrelevant. ${ }^{62}$ Es scheint nämlich, dass wir zumindest in mancher Hinsicht heute eine funktionale Umkehrung bisheriger Beschleunigungsfaktoren der Moderne festzustellen haben: Die Instanzen, die einst die Nationalstaaten ausmachten und durch ein Mindestmaß an sozialer Kohärenz (Staat, Armee, Bürokratie, Demokratie, politische Regelsetzungen usw.) ihre rasche Modernisierung ermöglichten, scheinen nunmehr die von der Globalisierung angestoßene soziale Beschleunigung abzubremsen, mag man sich darüber nun freuen (wie die Anhänger eines starken, die Verzerrungen des Marktes korrigierenden Staates) oder es bedauern (wie die Befürworter der Ansicht, die von der transnationalen ökonomisch-technischen Sphäre ausgehende soziale Beschleunigung würde vermittels der Produktion verteilbarer Reichtümer das moderne Autonomieprojekt gewährleisten). Damit lässt sich vernünftigerweise für die post- 
kosellecksche Proposition plädieren ${ }^{63}$, die darin besteht, die Geschichte der Moderne als Geschichte sozialer Beschleunigung ins Auge zu fassen und die ewigen Debatten über die "Postmoderne« (Lyotard, Latour, Haraway), die "flüchtige Moderne» (Bauman) ${ }^{64}$, die »zweite« oder "reflexive Moderne« (Beck) ${ }^{65}$ oder auch die "Hochmoderne" und "Spätmoderne" (Giddens) ${ }^{66}$ durch die Feinperiodisierung spürbarer Beschleunigungsschübe zu ersetzen. Angesichts dessen, dass man sich alles in allem manchmal zu Recht fragt, was diese Debatten eigentlich zum Gegenstand haben, wäre der $z u$ erhoffende empirische Gewinn eines solchen Wechsels der Problemstellung vielleicht nicht zu vernachlässigen.

\section{Das Ende der Erinnerungsmilieus oder die »Zweite Französische Revolution«}

Im ersten, einleitenden Absatz zu seinem Gesamtunternehmen der Lieux de mémoire, der Erinnerungsorte, schrieb Pierre Nora 1984:

»Beschleunigung der Geschichte. Jenseits der Metapher gilt es zu ermessen, was der Ausdruck bedeutet: ein immer rascheres Umkippen in eine definitiv abgestorbene Vergangenheit, die umfassende Wahrnehmung aller Dinge als verschwundene einen Bruch des Gleichgewichts. Das Herausreißen alles dessen, was noch gelebt wurde in der Wärme der Tradition, im Schweigen des Brauchtums, in der Wiederholung des Althergebrachten, angetrieben von einem tiefgreifenden historischen Gefühl. Erlangen des Selbstbewusstsein unter dem Vorzeichen des Überholten, Vollendung von etwas seit alters her Begonnenen. Von Erinnerung wird nur deshalb so viel geredet, weil es sie nicht mehr gibt. ${ }^{67}$

Und wenn es keine Erinnerungsmilieus mehr gibt und die Geschichte das Gefühl vermittelt, dass sie sich beschleunigt, so deswegen, weil zwischen 1965 und 1984 zumindest in Frankreich ein tiefer sozialer und zivilisatorischer Wandel eintrat, den Henri Mendras, der Begründer der französischen Agrarsoziologie, als »Zweite Französische Revolution« bezeichnete. ${ }^{68}$

Während dieser kurzen Zeitspanne ereignete sich nicht nur das "Ende der Bauern « ${ }^{69}$, sondern - über den radikalen Wandel des Bauerntums seit dem »Ende der Anbaugebiete ${ }^{70}$ hinaus - die Auflösung der drei anderen großen sozialen Klassen, die seit dem 19. Jahrhundert das gesamtgesellschaftliche System in Frankreich im Gleichgewicht hielten: des Proletariats, der Bourgeoisie und der »zwischen ihrer Herkunft aus niederen und bäuerlichen Schichten und ihrem bürgerlichen Ehrgeiz hin und her gerissenen « $^{71}$ Mittelklassen. Die an Langzeitperspektiven orientierten, natürlichen Rhythmen verpflichteten Milieus bäuerlicher Erinnerung sind untergegangen; die proletarischen Schichten haben sich im Verlauf der zunehmenden Tertiarisierung unserer Ökonomien zurückgebildet; die Bourgeoisie, durch Finanzkrisen und Inflation um ihre wirtschaftlichen Grundlagen gebracht, lebt heute wie jedermann »zunächst von ihrer Arbeit und nebenbei von ihrem Vermögen«; kurz: ein Klassensystem im ausgeprägten Sinn des Wortes ist innerhalb kurzer Zeit zusammengebrochen und hat einer Vielzahl sozialer Gruppen Platz gemacht, für die die überlieferte meritokratische Adäquanz von Einkommenshöhe und Ausbildungsabschlüssen absolut nicht mehr gilt. ${ }^{72}$

Die Identität der Individuen und sozialen Gruppen ist damit auf Dauer weitgehend zersplittert, und wenn man die tiefgreifenden Veränderungen im Bereich der Familie (Heirats-, Geburten-, Scheidungsquoten, neue Arbeitsteilung zwischen den 
Geschlechtern usw.) und die gewichtige Tatsache hinzunimmt, dass die herkömmlichen Garanten eines sozialen Mindestzusammenhalts (wie Kirche, Armee, Republik, Schule, Volksparteien und Massengewerkschaften) ihre symbolische Integrationsfunktion verloren haben, dann scheint alles dafür zu sprechen, dass angebrochen ist, was Mendras eine "neue Zivilisation der Sitten« mit noch ungewisser Zukunft nennt. Das Motiv der Beschleunigung der Geschichte erlaubt auch hier, dieses Ungewisse zu benennen, während die Inflation von Erinnerungsfeiern es ihrerseits unternimmt, es zu bannen.

\section{Das Ende der Moderne als innerweltlicher Verzeitlichung der Utopie}

51 Die Entstehung des »historischen Bewusstseins" in der zweiten Hälfte des 18. Jahrhunderts ging mit dem einher, was Koselleck als allgemeine Verzeitlichung der Geschichte und der Utopie bezeichnete. Diese Säkularisierung ${ }^{73}$ der traditionell religiösen Diskurse über die Hoffnung ließ den kollektiven Singular "Geschichte« als nicht nur ergänzenden, sondern zentralen »Faktor« einer Historizität erscheinen, der unterschiedlichen Akteuren erlaubt, ihr politisches Argumentieren und Handeln auf einer einzigen innerweltlichen Zeitskala $\mathrm{zu}$ situieren, die vorwärtsgewandt und unumkehrbar ist; damit entstand kraft einer unerhörten Überinvestition in die Zukunft und der zunehmenden Ideologisierung der politischen Projekte der moderne Perspektivismus.

Mit dem Fall der Berliner Mauer scheint dieses Zeitalter innerweltlicher verzeitlichter Ideologien vorübergehend an ein Ende gelangt, und das alte Thema der Beschleunigung der Geschichte zeigt sich heute nur mehr unter einer von jedem empirisch zuweisbaren telos abgekoppelten Doppelgestalt: als wirtschaftlich-technische Beschleunigung und als soziale Beschleunigung. Ein bestimmtes Regime des Glaubens an die Geschichte ist im Bewusstsein der modernen Menschen an sein Ende gelangt, und wir sind gewissermaßen »dazu verdammt, in dieser Welt zu leben ${ }^{74}$ Nicht dass die moderne demokratische Dynamik, d.h. die unaufhebbare Spannung zwischen dem Ideal der Freiheit und dem der Gleichheit, aufgehört hätte, ihre Wirkungen zu entfalten, und man sich nunmehr gemütlich in jenem »Ende der Geschichte« einrichten könnte, das sich angeblich in der repräsentativen Verfahrensdemokratie der entwickelten Länder verkörpert. ${ }^{75}$ Da die Zukunft sich jedoch nicht mehr zu einer krypto-parusischen Projektionsfläche eignet, scheint die Gegenwart sich zu entleeren und uns nur mehr dem Vakuum einer technischen und sozialen Beschleunigung ohne substanzielle wertspezifische Finalität näher zu bringen. Von diesem neuen Beschleunigungssystem ohne telos bleibt - ist uns der messianische Horizont der Geschichte erst einmal entzogen - nichts übrig als auf die Spitze getriebene Geschäftigkeit und Stress, Rastlosigkeit und Tyrannei des Zeitdrucks ${ }^{76}$, $\gg$ Präsentismus « ${ }^{77}$ und $» G e g e n w a r t s k u l t « .{ }^{78}$ Und dies ist heute sicher der gewichtigste Einwand gegen die Vorstellung einer kontinuierlichen Beschleunigung der Geschichte.

Definiert man die Moderne als Verzeitlichung der Utopie, dann ist die zeitgenössische Entteleologisierung tatsächlich so etwas wie das Totenglöckchen der Moderne und die "Auslöschung «"79 oder "kulturelle Entwertung der Zukunft" gleichbedeutend mit einer wahren anthropologischen Revolution, die vom "Perspektivmenschen" der

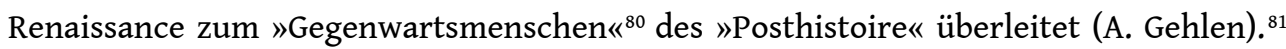
Der »Zeitdruck« liefe damit dem »Projekt« endgültig den Rang ab und die Heteronomie 
wirtschaftlicher und technisch-industrieller subsysteme dem modernen Ideal individueller wie kollektiver Autonomie.

\section{Zeitgenössische Strategien der »Entschleunigung»}

54 Doch gerade der Wunsch, das moderne Projekt des Autonomieideals aufrechtzuerhalten, steht hinter den meisten zeitgenössischen Diskursen über die notwendige »Entschleunigung" nicht mehr so sehr der Geschichte als vielmehr des zügellosen Tempos unserer Gesellschaften. Das Paradox besteht jedoch darin, dass dasselbe moderne Autonomieideal zu radikal unterschiedlichen politischen Optionen führt, die einander in der Wahl der Mittel zur Erreichung eines solchen gemeinsamen Ziels die Stirn bieten können. Die aus den Fugen geratene Zeit des Wirtschaftlichen und Sozialen $\mathrm{zu}$ »resynchronisieren ${ }^{82}$ mit den pluralischen Zeitrhythmen des individuellen Seelenlebens, der politischen Beratung und des ökologischen Gleichgewichts auf unserem Planeten: so lautet generell die Parole. Doch mehrere Varianten konkurrieren miteinander.

Diejenigen, für die der historische Kapitalismus und seine avancierte Form, die Globalisierung, den wichtigsten Motor einer heute unerträglich gewordenen sozialen Beschleunigung darstellen, zögern nicht, Strategien der "Zwangs-Re-Synchronisation« ${ }^{83} \mathrm{zu}$ predigen, um so die schädlichen Auswirkungen des technischen Fortschritts und der destruktiven Trägheit der Märkte rasch in den Griff $\mathrm{zu}$ bekommen. So sympathisieren manche altermondialistische ${ }^{84}$ oder radikalökologische Strömungen mit einer erzwungenen Entglobalisierung und Rezession unserer Wirtschaft, während eine breite, manchmal übertreibende, aber immer häufiger nuanciert argumentierende Literatur zu diesem Thema des früher oder später notwendigen »negativen Wachstums « der heutigen Welt floriert ${ }^{85}$ - und dies umso mehr, als die Menschheit des Industriezeitalters in 250 Jahren die Reserven an fossilen Brennstoffen aufgezehrt haben wird, die von der Natur in Jahrmillionen angehäuft wurden.

Andere, die das Prinzip der sozialen Marktwirtschaft als unzweifelhaftes Kriterium wirtschaftlicher Effizienz akzeptieren, setzen eher auf den Willen des Staates zur Durchsetzung politischer Anpassungsstrategien als Schlüssel zur erforderlichen Resynchronisation unserer Gesellschaften. Die Metapher vom Staat als "Herrn der Uhren « meint einen Staat, der mittel- und langfristig für das Gemeinwohl sorgt und allein in der Lage ist, sich um das Problem öffentlicher Einrichtungen, wichtiger strategischer Investitionen und externer Auswirkungen zu kümmern. Philippe Delmas unterstreicht in immer wieder neuen Variationen:

»Der Staat ist der Hüter der Uhren, der Beschaffer der erforderlichen Langsamkeit, dem Einfluss der Märkte entzogen, weil der Schnelligkeit konträr, die ihre Stärke ausmacht [...]. Um dauerhaft zur Verfügung zu stehen, muss das Kapital anderen Gesetzen gehorchen als den seinigen. Darin liegt der Sinn öffentlichen Handelns. Der Staat ist der Hüter der Uhren, dank dem die Zukunft die erforderliche Zeit hat heranzureifen. ¿ $^{86}$

Diese Metapher hat eine lange Geschichte, sie bringt die Gestalt des Sonnenkönigs als Prinzip absoluter, die Zeiten überdauernder Stabilität ins Spiel. Der Staat als »Herr der Uhren« unterscheidet sich in nichts vom Porträt des absolutistischen Monarchen als Chronokrator, als "Herr über die Zeit«, dessen Weisheit eine stabile Gegenwart gewährleistet, dessen Erinnerung eine intakte Vergangenheit garantiert und dessen Voraussicht uns eine dauerhafte, ungetrübte Zukunft sichert. ${ }^{87}$ Das Ideal der 
Beherrschung dieser unterschiedlichen Zeiten, das Gefahr läuft, die gerade aufgrund der ihnen aufgezwungenen technischen, wirtschaftlichen und sozialen Beschleunigung unterschiedliche Sphären unserer Gesellschaften auf Dauer zu entsynchronisieren, ist letzten Endes nichts anderes als die zeitgenössische demokratische (dem modernen regulativen Ideal der Autonomie und der Beherrschung des gemeinsamen Geschicks verpflichtete) Art und Weise, das der Metapher vom absolutistischen Chronokrator zugrunde liegende moderne Souveränitätsprinzip erneut zur Geltung zu bringen. Der demokratische Staat versucht, dem Markt auf gleicher Ebene zu begegnen: Er unternimmt es, die durch den Gang der Geschäfte bedingten unerwünschten Folgen von Geschwindigkeit, Flexibilität und permanentem Wandel zu konterkarieren, indem er sich zum Garanten des öffentlichen Interesses, nationaler Solidarität und dauerhafter sozialer Bindungen erklärt.

Aber der Wille zur Entschleunigung nimmt auch Formen an, die mit der Frage nach einer zentralen Staatsmacht und ihres Einsatzes politischer Strategien mit resynchronisierender Wirkung verhältnismäßig wenig $\mathrm{zu}$ tun haben. Gegen Produktivismus, Konsumismus und die gesellschaftliche Beschleunigung - überhaupt erst die Bedingung ihrer Möglichkeit - werden, ob individuell oder kollektiv, eine bessere Qualität subjektiver Lebenszeit und die Fähigkeit sozialer Akteure aufgewertet, humanere, weniger stressgeplagte Lebensformen $\mathrm{zu}$ erfinden; dahinter steht die Absicht, die Flut heterogener Ereignisse dem Bewusstsein psychologisch erträglich zu machen, so dass die Frage nach dem Sinn des Lebens selbst erneut und unter Absehung vom Leistungskult gestellt werden kann. Daraus entspringen eine Ästhetik und Ethik der »Langsamkeit « $^{88}$, unter deren Produkte die Regale der Buchhändler sich derzeit biegen, wobei die Gattungen munter durcheinanderschwirren (von auf hohem Niveau ästhetisierender Philosophie ${ }^{89}$ bis hin zu Tausenden hedonistischer Ratgeber für die eiligen Menschen, die wir sind und die erfahren sollen, wie sie ihre Zeit besser meistern und wirtschaftliche Effizienz endlich mit individuellem Glück verbinden können). ${ }^{90}$ Unter den philosophischen Diskursen über die unserer Zeit nottuende, existenzielle Langsamkeit erweist Walter Benjamin - mit seiner Thematik des messianischen Augenblicks (»Jetztzeit«), der das Kontinuum der Geschichte und der sozialen Beschleunigung mit einem Schlag zum Stillstand bringt - sich als besser geeignet als jeder andere, Herzen und Zungen zu lösen, was uns chronisch endlose Interpretationen der Geschichtsphilosophischen Thesen von $1940^{91}$ einbringt.

Eine letzte Art und Weise, die Thematik der »Entschleunigung" aufzugreifen, verdanken wir der kürzlich von Heinz Dieter Kittsteiner formulierten allgemeinen Diagnose der modernen Mentalität. ${ }^{92} \mathrm{Ihm}$ zufolge kommt es heute darauf an, in den ererbten politischen und kulturellen Praktiken tiefgreifend mit dem zu brechen, was er die »heroische Moderne« nennt, nämlich mit dem Willen, »Weltzeit« und "Lebenszeit» (Blumenberg) ${ }^{93}$ über den Aktivismus des Nietzscheschen Übermenschen oder des revolutionären Neuen Menschen zur Deckung zu bringen. Von diesem revolutionären Register heroischer Bemeisterung der Zeit, die Weltzeit und Lebenszeit zur Koinzidenz bringen möchte, habe man sich zu verabschieden - so folglich auch von dem linksradikalen Traum einer Zwangs-Re-Synchronisierung unserer Gesellschaften, mögen sie nun als historisch rückständig gelten (wie in den zahllosen tödlichen Aufrufen zum »Großen Sprung nach vorn«) oder als am Kult des Zeitdrucks erkrankt. Trotz zeitgenössischer radikaler, anti- oder altermondialistischer Versuchungen 
endgültig mit der politischen Figur der "Schwärmerei« zu brechen wäre demnach der letzte Schritt, den entschlossene Republikaner noch vor sich haben.

\section{BIBLIOGRAPHIE}

Agacinski, S. (2000): Le Passeur du temps. Modernité et nostalgie, Paris: Le Seuil.

Aubert, N. (2009): Le Culte de l'urgence: la société malade du temps, Paris: Flammarion.

Augustinus, A. (1985): Vom Gottesstaat, (De civitate dei), 2 Bde., übers. v. W. Thimme, München: dtv.

Bauman, Z. (2003): Flüchtige Moderne, übers. v. R. Kreissl, Frankfurt/M.: Suhrkamp

Beck, U. (2007): Weltrisikogesellschaft, Frankfurt/M.: Suhrkamp

Beck, U. / Lau, C. (2005): »Theorie und Empirie reflexiver Modernisierung. Von der

Notwendigkeit und den Schwierigkeiten, einen historischen Gesellschaftswandel innerhalb der

Moderne zu beobachten und zu begreifen«, Soziale Welt, 56 (2/3), S. 107-135.

Behringer, W. (2003): Im Zeichen des Merkur: Reichspost und Kommunikationsrevolution in der Frühen Neuzeit, Göttingen: Vandenhoeck \& Ruprecht.

Bengel, J. A.: Erklärte Offenbarung Johannis, Stuttgart: Erhardt, 1740.

Bengel, J. A.: Ordo temporum, Stuttgart: Erhardt, 1741.

Bengel, J. A.: Sechzig erbauliche Reden über die Offenbarung Johannis, Stuttgart: Erhardt, 1748.

Benjamin, W. (2010 [1942]): Über den Begriff der Geschichte, in: ders.: Werke und Nachlaß. Kritische Gesamtausgabe, Bd. 19, Berlin: Suhrkamp.

Benoist, J. / Merlini, F. (Hg.) (1999): Après la fin de l'histoire: temps, monde, historicité, Paris: Vrin.

Benz, E. (1973): Endzeiterwartung zwischen Ost und West. Studien zur christlichen Eschatologie, Freiburg i.B.: Rombach.

Benz , E (1977): Akzeleration der Zeit als geschichtliches und heilsgeschichtliches Problem, Mainz / Wiesbaden: Akademie der Wissenschaften und der Literatur / Steiner.

Blumenberg, Hans (2001[1986]): Lebenszeit und Weltzeit, Frankfurt/M.: Suhrkamp.

Bonstetten, C.-V. (1824): „L'Homme du Midi et l'homme du Nord«, in: Ce que nous avons été et ce que nous sommes - ou l'an 1789 et 1824 (Kap. XXIV), Genf / Paris: Paschoud.

Borscheid, P. (2004): Das Tempo-Virus. Eine Kulturgeschichte der Beschleunigung, Frankfurt/M.:

Campus.

Chateaubriand, F.-R. de (1831): Vorwort zu den »Etudes ou Discours historiques«, in: ders.: Cuvres complètes, Bd. IV, Paris: éd. Ladvocat.

Chateaubriand, F.-R. de (1968 [1849]): Erinnerungen. Mémoires d'outre-tombe, neu übers. u. hg. v.

S. v. Massenbach, Frankfurt/M. / Wien / Zürich: Büchergilde Guttenberg.

Corbin, A. (Hg.) (2009 [1995]): L'avènement des loisirs (1850-1960), Paris: Flammarion.

Delmas, Ph. (1992): Le Maître des horloges. Modernité de l'action politique, Paris: Odile Jacob. 
Delumeau, J. (1992, 1995, 2000): Une histoire du paradis, 3 Bde., Paris: Fayard.

Delumeau, J. (1998 [1978]): Angst im Abendland. Die Geschichte kollektiver Ängste im Europa des 14. bis 18. Jahrhunderts, übers. v. M. Hübner, Reinbek bei Hamburg: Rowohlt.

Desroche, H. (1969) (Hg.): Dieux d'hommes: Dictionnaire des messianisme et millénarismes de l'ère chrétienne, Paris / Den Haag: Mouton.

Escudier, A. (2006): „Pour une histoire européenne comparée des imaginaires historiographiques modernes du monde médiéval«, in: Fryde, N. / Oexle, O. G. / Monnet, P. (Hg.): L’Imaginaire moderne de la société médiévale (XIXe-XXIe siècles) / Die moderne Deutung der mittelalterlichen Gesellschaft (19.-21. Jahrhundert / Modern Conceptions of Medieval Society (19th.-21 Century), Göttingen: Vandenhoeck \& Ruprecht / Max Planck Institut für Geschichte, S. 381-423.

Flammarion, C. (1894): La fin du monde, Paris: Flammarion.

Fougier, E. (2004): Altermondialisme, le nouveau mouvement d'émancipation?, Paris: Lignes de repère.

Furet, F. (1996 [1995]): Das Ende der Illusion. Der Kommunismus im 20. Jahrhundert, übers. v.

K. Bartsch, E. Hagedorn, C. Krieger u. B. Reitz, München / Zürich: Piper.

Gehlen, A. (1971 [1963]): »Über kulturelle Kristallisation«, in: ders.: Studien zur Anthropologie und Soziologie, Neuwied / Berlin: Luchterhand (2. Aufl.), S. 311-328.

Gehlen, A. (1994): »Post-Histoire« (unveröff. Ms., hg. v. P. Tommissen), in: Klages, H. / Quaritsch, H. (Hg.) (1994): Karl Mannheim und die Krise des Historismus, Berlin: Duncker \& Humblot.

Giddens, A. (1996): Konsequenzen der Moderne, übers. v. J. Schulte, Frankfurt/M.: Suhrkamp.

Halévy, D. (2001): Essai sur l'accélération de l'histoire [1948], Le Quadrige emporté [1946], L'Histoire vat-elle plus vite? [1948], La Conquête des forces de la nature [1948], Leibniz et l'Europe [1940], hg. v. J.-

P. Halévy, Paris: Éd. de Fallois.

Hartog, F. (2003): Régimes d'historicité. Présentisme et expériences du temps, Paris: Le Seuil.

Heine, H. (1974 [1834]): Lutezia, zit. n. Heines Werke. Säkularausgabe, Bd. 11, Berlin / Paris:

Akademie-Verlag / Édition du CNRS.

Hölscher, L. (1989): Weltgericht oder Revolution. Protestantische und sozialistische

Zukunftsvorstellungen im deutschen Kaiserreich, Stuttgart: Klett-Cotta.

Hölscher, L. (1999): Die Entdeckung der Zukunft, Frankfurt/M.: Fischer.

Irenäus (1912): Des heiligen Irenäus fünf Bücher gegen die Häresien, übers. v. E. Klebba, München: Bibliothek der Kirchenväter, 1. Reihe, Bd. 3-4.

Jeanneney, J.-N. (2001): L'histoire va-t-elle plus vite? Variations sur un vertige, Paris: Gallimard.

Joutard, Ph. (1994 [1976]): Les camisards, Paris: Gallimard.

Jung-Stilling, J. H. (1994 [1794-1796]): Das Heimweh, hg. v. M. M. Sam. Dornach: Verlag am Goetheanum.

Jung-Stilling, J. H. (1799): Die Siegsgeschichte der christlichen Religion: in einer gemeinnützigen Erklärung der Offenbarung Johannis, Nürnberg: Raw.

Jurieu, P. (1994 [1686]): L’Accomplissement des prophéties, 2 Bde., Rotterdam: Abraham Acher, 1686; Reprint Bd. 2, eingeleitet von J. Delumeau, Paris: Imprimerie Nationale.

Justinus (1917): Dialog mit dem Juden Trypho, übers. v. Ph. Hauser, Kempten / München: Bibliothek der Kirchenväter, 1. Reihe, Bd. 33. 
Kaschuba, W. (2004): Die Überwindung der Distanz: Zeit und Raum in der europäischen Moderne, Frankfurt/M.: Fischer.

Kessel, M. (2001): Langeweile. Vom Umgang mit Zeit und Gefühlen in Deutschland vom späten 18. bis zum frühen 20. Jahrhundert, Göttingen: Wallstein.

Kittsteiner, H. D. (1999) (Hg.): Geschichtszeichen, Köln: Böhlau.

Kittsteiner, H. D. (2003): »Die Stufen der Moderne«, in: Rohbeck, J. / Nagl-Docekal, H. (Hg.): Geschichtsphilosophie und Kulturkritik. Historische und systematische Studien, Darmstadt: Wissenschaftliche Buchgesellschaft.

Klein, S. (2006): Zeit. Der Stoff aus dem das Leben ist. Eine Gebrauchsanleitung, Frankfurt/M.: Fischer.

Körtner, U. H. J. / Leutzsch, M. (Hg.) (1998 [1983]): Papiasfragmente, Darmstadt: Wissenschaftliche Buchgesellschaft.

Koselleck, R. (1998): »Geschichte« in Brunner, O. / Conze, W. / Koselleck, R. (Hg.): Geschichtliche Grundbegriffe, Bd. 2, Stuttgart: Klett-Cotta.

Koselleck, R. (2000): Zeitschichten. Studien zur Historik, Frankfurt/M: Suhrkamp.

Koselleck, R. (2000a), »Gibt es eine Beschleunigung der Geschichte?« [1976], in: Koselleck (2000), S. 150-176.

Koselleck, R., (2000b): »Zeitverkürzung und Beschleunigung. Eine Studie zur Säkularisation « [1985], in: Koselleck (2000), S. 177-202.

Koselleck, R. (2000c): »Die Verzeitlichung der Utopie« [1982], in: Kosellek (2000), S. 131-149.

Koselleck, R. (2000d): »Historik und Hermeneutik« [1985], in: Koselleck (2000), S. 97-118.

Laïdi, Z. (1998): „L'urgence et la dévalorisation de l'avenir«, Esprit, Februar, S. 8-20.

Laïdi, Z. (1999): La Tyrannie de l'urgence, Montreal, Fides/Quebec: Musée de la civilisation.

Laïdi, Z. (2000): Le Sacre du présent, Paris: Le Seuil.

Lassalle, F. (1990): Franz von Sickingen, Stuttgart: Metzler.

Laube, R. (2003): Karl Mannheim und die Krise des Historismus: Historismus als wissenssoziologischer Perspektivismus, Göttingen: Vandenhoeck \& Ruprecht.

Lecourt, D. (2007 [1993]): Contre la peur: de la science à l'éthique, une aventure infinie, Paris: PUF.

Le Goff, J. (1984): „Le Moyen Âge entre le futur et l'avenir«, Vingtième siècle, 1 (1), S. 15-22.

Löper, C. (Hg.) (1881): Stammbuch der neueren Verkehrsmittel. Eisenbahnen, Dampfschiffe, Telegraphen und Luftschiffe. Eine Sammlung von Liedern und Gedichten, Lahr: Schauenburg.

Löwith, K. (2004 [1949]): Weltgeschichte und Heilsgeschehen. Die theologischen Voraussetzungen der Geschichtsphilosophie, Stuttgart: Metzler.

Luther, M. (1912 [1534]): Die Bibel oder die ganze Heilige Schrift, Stuttgart: Privilegierte Württembergische Bibelanstalt.

Maurice, K. (1967): Die französische Pendule des 18. Jahrhunderts. Ein Beitrag zu ihrer Ikonologie, Berlin: de Gruyter.

Mendras, H. (1992 [1967]): La fin des paysans: changements et innovations dans les sociétés rurales françaises, erweiterte Ausg., Le Paradou / Paris: Actes Sud. 
Mendras, H. (1994 [1988]): La Seconde Révolution française (1965-1984), überarbeitete Ausg., Paris: Gallimard.

Mercier, L.-S. (1982 [1771]): Das Jahr 2440. Ein Traum aller Träume, übers. v. C. F. Weiße, Frankfurt/ M.: Insel.

Monod, J.-C. (2002): La Querelle de la sécularisation, Paris: Vrin.

Nadolny, Sten (2010 [1983]): Die Entdeckung der Langsamkeit, München: Piper.

Niethammer, L. (1989): Posthistoire. Ist die Geschichte zu Ende?, Reinbek: Rowohlt.

Nora, P. (Hg.) (1997[1984]): »Entre Mémoire et Histoire. La problématique des lieux«, in: ders.: Les Lieux de mémoire, Paris: Gallimard, Bd. 1, S. 23-43.

Nowotny, H. (1959): Eigenzeit. Entstehung und Strukturierung eines Zeitgefühls, Frankfurt/M.:

Suhrkamp.

Ollivro, J. (2007): Quand la vitesse change le monde: essor de la vitesse et transformation des sociétés, Rennes: Apogée.

Rabanne, P. (1996 [1993]): Das Ende unserer Zeit, Aufbruch in das Wassermannzeitalter. Prophezeiungen des neuen Nostradamus, übers. v. G. Meier, München: Droemer Knaur.

Radkau, J. (1998): Das Zeitalter der Nervosität: Deutschland zwischen Bismarck und Hitler, München: Hanser.

Rifkin, J. (1990 [1987]): Uhrwerk Universum. Die Zeit als Grundkonflikt des Menschen, übers. v. M. Huber, München: Droemer Knaur.

Rosa, H. (2003): „Social Acceleration: Ethical and Political Consequences of a Desynchronized High-Speed Society, Constellations. An international Journal of Critical and Democratic Theory, 10 (1), März, S. 3-33.

Rosa, H. (2010): Beschleunigung. Die Veränderung der Zeitstruktur in der Moderne, Frankfurt/M.: Suhrkamp.

Rosa, H. / Clemens, J. / Mayer, M. (Hg.) (2004): Fast forward. Essays zu Zeit und Beschleunigung. Standpunkte junger Forschung, Hamburg: Edition Körber-Stiftung.

Salmon, J.-M. (2000): Un monde à grande vitesse. Globalisation, mode emploi, Paris: Le Seuil.

Schivelbusch, W. (1977): Geschichte der Eisenbahnreise: Zur Industrialisierung von Raum und Zeit im 19. Jahrhundert, München: Hanser.

Schmitt, C. (2009 [1922]): Politische Theologie. Vier Kapitel zur Lehre von der Souveränität, Berlin: Duncker \& Humblot.

Simmel, G. (1992 [1897-1900]): «Die Bedeutung des Geldes für das Tempo des Lebens«, in: ders. Gesamtausgabe, Bd. 5: Aufsätze und Abhandlungen 1894 bis 1900, Frankfurt/M.: Suhrkamp, S. 215-234.

Taguieff, P.-A. (2000): L'Effacement de l'avenir, Paris: Galilée.

Taguieff, P.-A. (2001): Résister au bougisme: démocratie forte contre mondialisation techno-marchande, Paris: Mille et une nuits.

Valéry, P. (1995 [1938]): »Fluktuationen über die Freiheit«, in: ders. Werke, Bd. 7, hg. v. J. SchmidtRadefeldt, Frankfurt/M. / Leipzig: Insel Verlag, S. 295-316.

Weber, E. (1983): La Fin des terroirs: la modernisation de la France rurale (1870-1914), Paris: Fayard. 


\section{NOTES}

1. Valéry (1995), S. $315 \mathrm{f}$.

2. Koselleck (2000 a, b).

3. Borscheid (2004).

4. Rosa (2003, 2010); Rosa / Clemens / Mayer (Hg.) (2004).

5. Die eschatologische, aber antichiliastische Einstellung des Heiligen Augustinus (Vom Gottesstaat XX, $7 \mathrm{f}$.) spielte für die Herausbildung der antimillenaristischen Position der institutionalisierten Kirche eine beträchtliche Rolle. Innerhalb der eschatologischen Diskurse ist daher die antichiliastische eschatologische Positionierung der Kirche (angefangen bei Eusebius von Caesarea und seiner Kirchengeschichte [III, XXXIX, 11-13 und XII, XXXIX, 31a], sodann Augustinus) von den millenaristischen Eschatologien nicht durch die offizielle Kirche ermächtigter Individuen und Gruppen durchaus zu unterscheiden, denen selbstverständlich der Schwefelgestank der Revolution oder zuallermindest des sozialen Chaos anhing. Vgl. dazu Delumeau (1995), S. 30 f. und passim.

6. Abgesehen von einigen zeitgenössischen neochiliastischen Diskursen innerhalb mancher Sekten oder bei vereinzelten Autoren wie Paco Rabanne (1996).

7. Matthäus XXIV, 36; Jurieu (1994 [1686]), Bd. 2, Kap. XXIII, S. 308.

8. Obschon es hier nicht möglich ist, auf die unterschiedlichen eschatologischen Varianten einzugehen, können die starken Disparitäten unter ihnen doch nicht ganz außer Acht bleiben. So muss die vom Heiligen Augustinus ausgehende Überlieferung stark von der Lehre von den drei Weltzeitaltern Joachim von Fiores unterschieden werden, desgleichen von dem radikalen und militanten Chiliasmus der böhmischen Taboriten und später der Münsteraner Wiedertäufer. Vgl. zu all dem die von Jean Delumeau in seiner ersten Untersuchung (1998 [1978]) und vor allem in ihrer sehr viel eingehenderen Weiterführung (1992, 1995, 2000) eingeführten, idealtypischen Unterscheidungen; vgl. vor allem Delumeau (1995).

9. Bengel $(1740,1741,1748)$.

10. Jung-Stilling 1994 [1794-1796], 1799.

11. Matthäus XXIV, 36.

12. Übersetzt von Martin Luther (1912 [1534]). [A.d.Ü.]

13. Matthäus XXIV, 46 .

14. Matthäus XXIV, 36 .

15. Geheime Offenbarung XII, 12.

16. Vgl. Augustinus, Vom Gottesstaat, XX, Kap. 8, und Jurieu (1994 [1686]), Kap. XXII, S. 300 f.

17. $\mathrm{Zu}$ der entsprechenden Literatur vgl. Benz $(1973,1977)$. In anderen Zusammenhängen predigten manche religiöse Gruppen die Auswanderung in die Neue Welt, deren Zukunft entweder als völlig unbestimmt und offen galt oder das dritte Weltzeitalter, das des Geistes, darstellen sollte, das der Parusie und dem eigentlichen Ende der Welt um tausend Jahre vorgeschaltet war. Vgl. Delumeau (1995), S. 275 f.

18. Jurieu (1994).

19. Jurieu (1994), S. 65 und $300 \mathrm{f}$.

20. Vgl. die klassischen Arbeiten von Philippe Joutard (1994 [1976]), S. 64 f.

21. Justinus (1917).

22. Körtner / Leutzsch (1998).

23. Irenäus (1912). Zum Millenarismus der apostolischen Väter und späterer Zeiten vgl. die beiden klassischen Arbeiten von Henri Desroche (1969) und J. Delumeau (1995).

24. Jurieu (1994 [1686]), Kap. XXIII, S. 303 f. Noch am Ende des 19. Jahrhunderts setzt Camille Flammarion (1894, S. 166) den Begriff »millénaires« für die Millenaristen ein. 
25. Zur allmählichen Transformation religiöser Kategorien der Erwartung in Agenda sozialistischer Hoffnung während der zweiten Hälfte des 19. Jahrhunderts vgl. die Untersuchung von Lucian Hölscher (1989).

26. Lassalle (1990).

27. Vgl. Kosellecks klassische Studie »Geschichte« in: Koselleck (1998).

28. Vgl. zu diesem Punkt die Arbeiten Lucian Hölschers (1999). Jaques Le Goff (1984) macht eine Nuancierung dieses Befunds möglich, freilich auf der Grundlage einer Mentatlitätengeschichte und keineswegs auf der Ebene der historischen Semantik.

29. Matthäus XXIV.

30. Diese Unterscheidung zwischen künftigen Dingen und Zukunft ist von Wichtigkeit, denn es wäre absurd, auf die historische Semantik gestützt zu behaupten, die Vorstellung künftiger Ereignisse (im Unterschied zu gegenwärtigen oder vergangenen) habe vor dem 18. Jahrhundert nicht existiert. Selbstverständlich geht es hier um etwas ganz anderes, nämlich um die Geschichte im Verlauf der Zeitalter wechselnder »Erwartungshorizonte«.

31. Halévy (2001), S. 66, 102, 154.

32. Koselleck (2000c).

33. Koselleck (1998).

34. Bonstetten (1824), S. 203. Doris und Peter Walser-Wilhelm, den Herausgebern der bewundernswerten Bonstettiana (Göttingen: Wallstein, 1996 ff.), bin ich für diesen Hinweis sehr zu Dank verpflichtet. [A.d.Ü.: Die im Rahmen dieser Reihe erscheinenden Spätschriften 1810-1826, die die zitierte Passage enthalten, waren zum Zeitpunkt der Übersetzung noch nicht greifbar.]

35. Chateaubriand (1831), S. CXXXVI f. (Hervorhebung v. A. E.).

36. Chateaubriand, F.-R (1968 [1849]), S.732 f. (Hervorhebung v. A. E.)

37. Chateaubriand, F.-R (1968 [1849]), S. 746.

38. Man erlaube mir den Hinweis auf eine Studie, in der ich versucht habe, diesen Punkt im Hinblick auf die Phantasievorstellungen der Moderne vom Mittelalter zu formalisieren: Escudier (2006).

39. Diese Interpretationsmöglichkeit wurde kürzlich von Kittsteiner (1999) erschlossen.

40. Zur Geschichte der nicht zu vernachlässigenden, da organisationsbedingten, qualitativen Sprünge innerhalb ein und desselben technischen Zeitalters vgl. die kürzlich von Behringer (2003) erarbeitete Synthese.

41. Vgl. Schivelbusch (1977) und Kaschuba (2004).

42. »Eisenbahnen reduzieren Europa ungefähr auf den Flächenraum Deutschlands«, siehe das Stichwort "Eisenbahnen« im Conversations-Lexikon der Gegenwart in vier Bänden, Leipzig: Brockhaus, 1838, S. 1115-1136; hier zitiert nach Koselleck (2000a), S. 160.

43. Heine (1974 [1834]), S. 181f. Zahlreiche ähnliche Zeugnisse finden sich bei Löper (1881).

44. Louis Sébastien Mercier in seinemantizipatorischen Roman von 1771: Das Jahr 2440 (1982), S. 27.

45. Chateaubriand (1968 [1849]), S. 733.

46. Vgl. Heine (1974 [1834]), S. 182: »Durch die Eisenbahnen wird der Raum getödtet, und es bleibt uns nur noch die Zeit übrig. Hätten wir nur Geld genug, um auch letztere anständig zu tödten!«

47. $\mathrm{Zu}$ den unterschiedlichen Bestandteilen dieser noch im Aufbau begriffenen Kulturgeschichte vgl. Corbin (2001).

48. Kessel (2001).

49. Vgl. dazu Salmon (2000).

50. Simmel (1992 [1897-1900]), S. 217.

51. Ollivro (2007).

52. Radkau (1998).

53. Vgl. die Untersuchungen von Pierre-André Taguieff (2001). 
54. Vgl. Salmon (2000).

55. $\mathrm{Zu}$ der »quasi transzendentalen« Kategorie "geheim« als Bedingung der Möglichkeit aller Politik und also der wirklichen Geschichte selbst vgl. Kosellecks inzwischen klassischen Beitrag "Historik und Hermeneutik« (2000 d).

56. Vgl. zum Beispiel die Erwiderung auf Jonas und den Heidelberger Aufruf bei Dominique Lecourt (1993).

57. Beck (2007).

58. Aron (1970 [1968]), S. 276.

59. Vgl. Scheuerman $(2001,2004)$.

60. Dieser Artikel der französischen Verfassung ermöglicht der Regierung, eine parlamentarische Abstimmung mit der Vertrauensfrage zu verbinden und damit die Zustimmung aller Abgeordneten zu erzwingen, die einen Sturz der Regierung vermeiden wollen. [A.d.Ü.]

61. Vgl. z. B. Jean-Noël Jeanneneys Kommentar (2001, S. 120 f.) zu den kritischen Äußerungen Michel Rocards.

62. Rosa (2010), S. 311-329, $402 \mathrm{f}$.

63. Wobei festzuhalten bleibt, dass diese These nur die soziologisierende Rückübersetzung des von R. Koselleck seit dem letzten Drittel des 18. Jahrhunderts ausgemachten »Verzeitlichung der Geschichte« ist.

64. Bauman (2003)

65. Zu der Kritik am Begriff „zweite Moderne“ vgl. die Klarstellung von Beck / Lau (2005).

66. Giddens (1996).

67. Nora (1997[1984]).

68. Mendras (1994).

69. Mendras (1992).

70. Weber (1983).

71. Mendras (1994), S. 29.

72. Mendras (1994), S. 29.

73. In diesem Punkt ist R. Koselleck der klassischen Untersuchung Karl Löwiths (2004 [1949]) mindestens ebenso verpflichtet wie der frühen politischen Theologie Carl Schmitts (2009 [1922]). Vgl. außerdem das treffliche Werk von Jean-Claude Monod (2002).

74. Furet (1996 [1995]), S. 覧[5:[59.

75. Benoist / Merlini (Hg.) (1999).

76. Aubert (2009).

77. Taguieff (2000), S. 95 f.; Hartog (2003).

78. Vgl. Laïdi (1998, 1999, 2000).

79. Taguieff (2000).

80. Dies ist die zentrale These Zaki Laïdis, der damit unmittelbar an die Untersuchungen R. Kosellecks anschließt (wobei Laïdi allerdings die zahlreichen historischen und theoretischen Vermittlungen überspringt, die zwischen dem pikturalen Perspektivismus der Renaissance und dem verzeitlichten Perspektivismus der Geschichtsphilosophen aus der zweiten Hälfte des 18. Jahrhunderts bestanden haben). In dieser Hinsicht gab Koselleck selbst lediglich der europäischen Debatte über die »Krise des Historismus« nach 1914-1918 (wie Mannheim sie ab 1924 unter dem Vorzeichen eines dem modernen Menschen eigenen, historistischen Perspektivismus reinterpretiert hatte) eine Wendung $\mathrm{zu}$ einer Theorie der historischen Verzeitlichungen und zu einer Kritik der politischen Soziodizeen. Vgl. dazu die umfassende Untersuchung von Reinhard Laube (2003).

81. Gehlen (1971 [1963], 1994). Vgl. auch Niethammer (1998).

82. Rifkin (1990).

83. Rosa (2010), S. 488.

84. Vgl. die von Eddy Fougier (2004) unterschiedenen fünf Strömungen. 
85. Vgl. z. B. die Zeitschrift Entropia. Revue d'étude théorique et politique de la décroissance, Lyon: Parangon, 2006ff.

86. Delmas (1992), S. 27 und 83 (Hervorhebung durch A. E.).

87. Vgl. Maurice (1967).

88. Man denke an den Welterfolg des pseudohistorischen Romans, den der deutsche Schriftsteller Sten Nadolny (2010 [1983]) über den Kapitän und Polarforscher John Franklin (1786-1847) schrieb.

89. Vgl. z. B. Nowotny (2010 [1989]) und Agacinski (2000).

90. Vgl. u. a. den Schlussteil des Buchs von Stefan Klein (2006).

91. Benjamin (2010 [1942]).

92. Vgl. Kittsteiner (1999 und 2003).

93. Blumenberg (2001 [1986]).

INDEX

Mots-clés : accélération de l'histoire, décélération, millénarisme, révolution, zukunft, temporalisation

Schlüsselwörter : Beschleunigung der Geschichte, Entschleunigung, Millenarismus, Revolution, Zukunft, Verzeitlichung

\section{AUTEURS}

\section{ALEXANDRE ESCUDIER}

Alexandre Escudier (geb. 1971) ist wissenschaftlicher Mitarbeiter am Centre de Recherches Politiques de Sciences Po (CEVIPOV, Paris). Nähere Informationen finden Sie hier. 